\title{
Testing general relativity during the cruise phase of the BepiColombo mission to Mercury
}

\author{
Luigi Imperi \\ Departement of Mechanical and \\ Aerospace Engineering \\ Sapienza University of Rome \\ Rome, Italy \\ Email: luigi.imperi@uniroma1.it
}

\author{
Luciano Iess \\ Departement of Mechanical and \\ Aerospace Engineering \\ Sapienza University of Rome \\ Rome, Italy \\ Email: luciano.iess@uniroma1.it
}

\begin{abstract}
General relativity (GR) predicts that photons are delayed and deflected by the space curvature produced by any mass. The Post-Newtonian (PN) parameter controlling the curvature induced by a gravitational field is $\gamma$, with bending and delay effects proportional to $(\gamma+1) \cdot \gamma=1$ in GR. The most accurate estimation of this PN parameter $\gamma=(1+(2.1 \pm 2.3)) \cdot 10^{-5}$, has been obtained by the NASA mission Cassini [1] exploiting the frequency shift of radio signal during a Superior Solar Conjunction (SSC) in 2002, while the spacecraft was in cruise to Saturn. The crucial element of the experiment was an advanced radio system providing a highly stable multi-frequency radio link in $\mathrm{X}$ and $\mathrm{Ka}$ band $(8.4$ and $32.5 \mathrm{GHz})$, and a nearly complete cancellation of the plasma noise introduced by the solar corona in Doppler measurements.

The ESA-JAXA mission BepiColombo to Mercury will improve the Cassini radio instrumentation by enabling the ranging function also in the Ka band radio link used by the Mercury Orbiter Radio science Experiment (MORE). The fully digital architecture of the transponder provides a pseudo-noie modulation of the carrier at 24 Mcps and a two-way range accuracy of $20 \mathrm{~cm}$. Thanks to the simultaneous tracking by means of the standard telecommunication link, both range and range rate observables will be available for new, more accurate tests of GR. This paper reports on the simulations carried out in order to assess the attainable accuracies in the estimation of $\gamma$ during the cruise phase of BepiColombo. In an optimal configuration, an uncertainty of $5 \cdot 10^{-6}$ may be attained.
\end{abstract}

Keywords-Radio Science, General Relativity, Mercury, BepiColombo, Superior Conjunction

\section{INTRODUCTION}

\section{A. Motivations}

General Relativity (GR), published by Albert Einstein in 1915 as a generalization of special relativity, changed dramatically our understanding of space and time and is a cornerstone of our description of the physical world. Many predictions of GR have been experimentally tested, making it one of the most successful physical theories. General relativity is not only a more accurate theory of gravity, but also a tool to investigate the phenomena occurring in remote recesses of the universe. Indeed, gravitational wawes, predicted by GR but not yet observed, will shed light on the processes occurring in active galactic nuclei, hidden to observations in the electromagnetic spectrum.

However, this powerful theory is incompatible with quantum mechanics. Many efforts have been devoted to the development of a "theory of everything", able to unify all fundamental forces of nature, including gravity. Such a unification will almost certainly require changes in GR. General relativity itself is only the simplest among a family of theories which are equally plausible on theoretical grounds. However, the experimental evidence gained so far makes those alternative theories very similar to GR. In any case, all alternative theories depart from GR at some level of experimental accuracy. Hence, it is of crucial importance to assess at what level it may be violated.

\section{B. The BepiColombo Radio science Experiment}

BepiColombo is a dual spacecraft mission jointly developed by ESA (European Space Agency) and JAXA (Japan Aerospace and Exploration Agency) and devoted to a comprehensive study of the planet Mercury. The Mercury Magnetosphere Orbiter (MMO), provided by JAXA, will study the exosphere and magnetosphere of Mercury, while the ESA's Mercury Planetary Orbiter (MPO), placed in a lower, nearly circular orbit around the planet, will study the characteristic of the surface and the deep interior.

The Mercury Orbiter Radio science Experiment (MORE) of the MPO consists of ground and onboard instrumentation enabling a highly stable, multi-frequency radio link at $\mathrm{X}$ and $\mathrm{Ka}$ band. Range rate measurements obtained from this advanced radio link will be unaffected by plasma noise and are expected to attain accuracies of $3 \mathrm{micron} / \mathrm{s}$ (at 1000 seconds integration time) at nearly all elongation angles. Thanks to a novel wideband ranging system, based on a 24 Mcps pseudo-noise modulation, the spacecraft range will be measured to an accuracy of $20 \mathrm{~cm}$ (two-way). The MORE investigation will also greatly benefit from a direct measurement of the vectorial non-gravitational accelerations by means of the Italian Spring Accelerometer (ISA).

The high quality radio-metric observables will provide a precise reconstruction of the spacecraft orbit and an accurate estimation of the gravity field and rotational state of the planet. In addition, BepiColombo will carry out tests of GR by reconstructing the orbit of the planet and the propagation of photons in the solar gravitational field. Indeed, since the orbit of Mercury is affected more than any other 


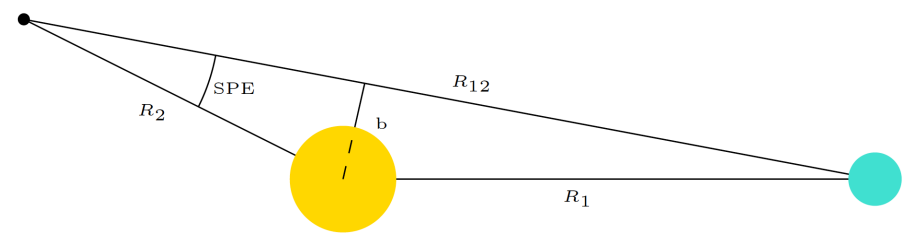

Fig. 1. Solar superior conjunction geometry

planet by relativistic effects, MORE aims at improving the determination of several Post-Newtonian (PN) parameters: $\beta, \gamma$, the Nordtvedt parameter $\eta$ and the preferred frame parameters $\alpha_{1}, \alpha_{2}$ [2]. Further physical parameters such as the rate of change of the gravitational parameter and the oblateness factor $J_{2}$ of the Sun will be estimated as well.

\section{THE SUPERIOR SOLAR CONJUNCTION EXPERIMENT}

\section{A. The Shapiro delay}

According to GR, photons are deflected and delayed by a gravity field. While the first effect was already found by Einstein, the time delay was discovered only in 1964 by Irwin I. Shapiro [3]. Following Will [4] and Moyer [5], the radio delay, known as Shapiro delay, on a single leg signal is:

$$
\Delta t=\frac{(1+\gamma) G M}{c^{3}} \ln \left(\frac{R_{1}+R_{2}+R_{12}}{R_{1}+R_{2}-R_{12}}\right)
$$

where $\mathrm{G}$ is the gravitational constant, $\mathrm{c}$ the velocity of light and $\mathrm{M}$ the mass of the body producing the space-time curvature. $R_{1}, R_{2}$ and $R_{12}$ are respectively the positions of the sender, the receiver and their mutual distance expressed in a system whose origin is in the center of mass of the body (see Figure 1). The value of $\gamma$, which measures the space curvature induced by unit mass, is unity in GR.

During a superior conjunction $R_{1}+R_{2} \approx R_{12}$ and the effect is strongly enhanced. In this case, (1) can be approximated, (b $\left.<<R_{1}, R_{2}\right)$ as [1]:

$$
\Delta t=\frac{(1+\gamma) G M}{c^{3}} \ln \left(\frac{R_{1} R_{2}}{b^{2}}\right)
$$

\begin{tabular}{|c|c|c|c|c|c|}
\hline SSC & Date & b min & $\begin{array}{c}\text { AU from } \\
\text { the Sun }\end{array}$ & $\begin{array}{c}\text { S-E } \\
\text { propulsion }\end{array}$ & Notes \\
\hline $\mathbf{I}$ & 28 AUG 2019 & 2.24 & 0.60 & No & $\begin{array}{c}\text { just before a } \\
\text { Venus flyby }\end{array}$ \\
\hline II & 19 AUG 2020 & 8.30 & 0.44 & Yes & - \\
\hline III & 18 APR 2021 & 1.85 & 0.32 & No & $\begin{array}{c}\text { includes a } \\
\text { Mercury flyby }\end{array}$ \\
\hline IV & 30 AUG 2021 & 7.71 & 0.39 & Yes & - \\
\hline $\mathbf{V}$ & 09 MAR 2022 & 7.28 & 0.38 & Yes & - \\
\hline VI & 16 JUL 2022 & 7.99 & 0.31 & No & $\begin{array}{l}\text { includes a } \\
\text { Mercury flyby }\end{array}$ \\
\hline VII & 08 NOV 2022 & 0.57 & 0.44 & Yes & - \\
\hline VIII & 14 MAR 2023 & 5.57 & 0.35 & Yes & - \\
\hline IX & 28 JUN 2023 & 4.68 & 0.31 & Yes & - \\
\hline $\mathbf{X}$ & 19 OCT 2023 & 3.03 & 0.43 & Yes & - \\
\hline
\end{tabular}

TABLE I. DATA OF THE CONJUNCTIONS

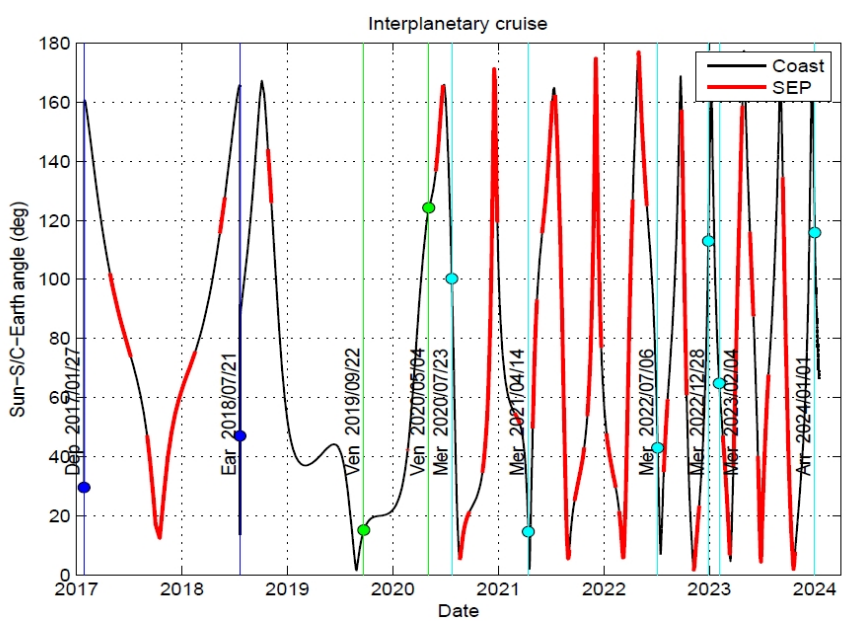

Fig. 2. Sun-Probe-Earth angle during the cruise phase (from [8]). The red thick lines indicate arcs with solar-electric propulsion (SEP), while colored marker indicate flybys

where $b$ is the impact parameter ${ }^{1}$ (Figure 1). The corresponding frequency shift $\Delta \nu / \nu$ is [6]:

$$
\frac{d \Delta t}{d t}=\frac{(1+\gamma) G M}{c^{3} b} \frac{d b}{d t}
$$

The Doppler shift can be also be viewed as a consequence of the gravitational deflection of the radio beam [6].

The Shapiro delay is directly observable by means of range measurements (2), but also through the Doppler shift (3) (see Figure 3). As a consequence, a Superior Solar Conjunction (SSC) is an opportunity to test GR by using both types of radio-metric observables.

\section{B. The Cassini experiment and the BepiColombo opportunity}

The relativistic Doppler shift provided an accurate test of GR. It was carried out by the mission Cassini (NASA, ESA and ASI) during the cruise phase to the Saturn system. In June 2002 a SSC of the Cassini spacecraft allowed to determine the value of $\gamma$ as $(1+(2.1 \pm 2.3)) \cdot 10^{-5}$ by means of Doppler measurements only. The use of the relativistic Doppler shift for testing GR did not take place for many years since the beginning of solar system exploration, the main reason being the overwhelming noise contribution due to the solar corona. The Cassini mission overcame the problem by adopting high frequency microwave links at Ka-band in addition to the $\mathrm{X}$-band link used for standard spacecraft operations. Since plasma is a dispersive medium, the use of carriers at different frequencies (multi-frequency radio links) provides the path delay (for range measurements) and its time derivative (for Doppler measurements) caused by the intervening plasmas (solar corona, interplanetary plasma and ionosphere). In the limit of geometric optics, plasma noise can be completely cancelled out $[7,13]$.

Enhabling multi-frequency radio links requires dedicated instrumentations both onboard and at the ground antennas. In the Cassini and BepiColombo radio science experiments,

\footnotetext{
${ }^{1}$ In this work we always refer to $\mathrm{b}$ as expressed in solar radii
} 

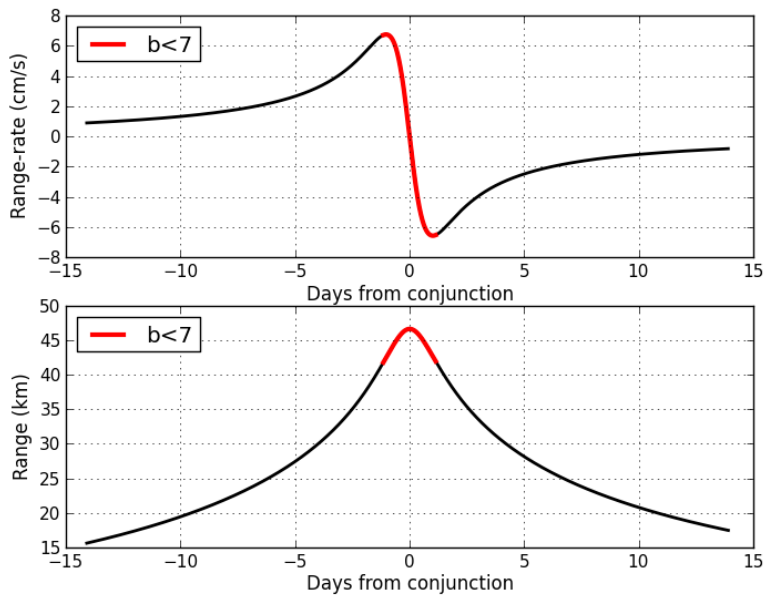

Fig. 3. Time delay effect on two-way measurements for the ninth superior conjunction $(\gamma=1)$. Red lines represent when $\mathrm{b}<7$, which is a reasonable limit for the applicability of the plasma noise compensation procedure

the spacecraft hosts a dedicated transponder capable of receiving an uplink at $\mathrm{Ka}$ band $(34 \mathrm{GHz})$ and retransmitting it coherently to ground (at $32.5 \mathrm{GHz}$ ). The supporting ground antenna (DSS 25 of NASA's Deep Space Network, located in Goldstone, California) is equipped with a Ka band transmitter and additional instrumentations for precise antenna pointing and the calibration of tropospheric delays. Recently, ESA has successfully tested $\mathrm{Ka}$ band transmit capabilities at its Malargue (Argentina) Deep Space Antenna (DSA 3) and is planning to reach full operational capabilities to support the MORE investigation, in addition to DSS 25. Remarkably, BepiColombo's MORE Ka band transponder (KaT) enables not only Doppler measurements (as in the case of Cassini), but also range measurements.

The description of the BepiColombo cruise phase is given in [8]. The mission has several opportunities to carry out GR tests of time delay during its long cruise phase to Mercury, potentially improving over Cassini's result. In the current baseline option, launch is scheduled on January 27, $2017^{2}$ with arrival at Mercury on January 1, 2024, after one Earth flyby, two Venus flybys and five Mercury flybys. Figure 2 shows the Sun-Probe-Earth (SPE) angle over the whole cruise trajectory. There are ten potentially exploitable SSC starting from August 2019. Table 1 reports the main characteristics of the conjunctions, only during the seventh one (November 2022) BepiColombo will be occulted by the solar disk.

As BepiColombo uses a solar-electric propulsion system over large segments of the cruise, not all SSC can be exploited for the GR test, which requires the spacecraft to be in a quiet state. Currently only the first, the third and the sixth SSC, occurring close to a planetary flyby, correspond to coast arcs.

\footnotetext{
${ }^{2}$ There are two backup launch options in 2017, however the geometry of the SSC does not change appreciably
}
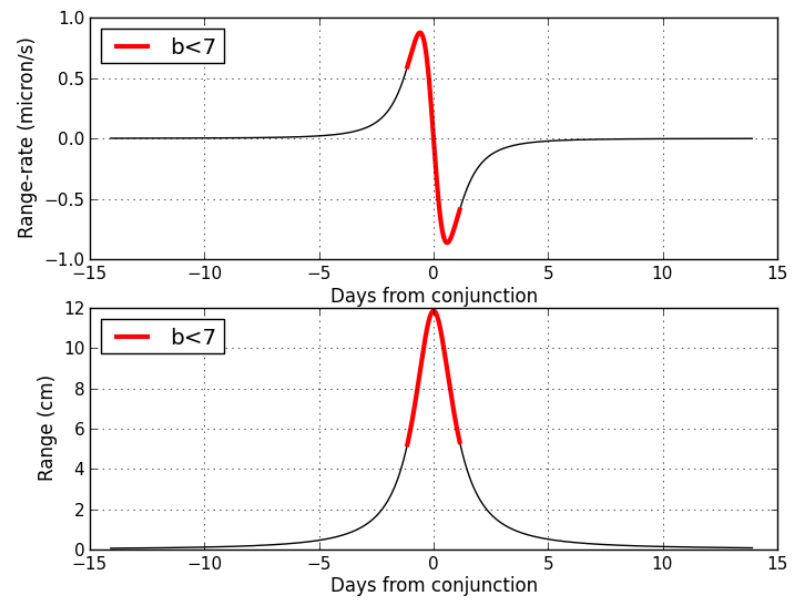

Fig. 4. Effect of 2-PN level correction on two-ways measurements for the ninth superior conjunction $(\gamma=1)$. Red lines represent when $\mathrm{b}<7$. The correction becomes relevant if data below this value of the impact parameter were available (references [9] and [10] assume the data to be avalilble down to three solar radii)

\section{NUMERICAL SIMULATIONS}

\section{A. Assumptions}

Equation (1) holds to the 1-PN level (i.e. to the $\left.(v / c)^{2}\right)$. As discussed in [9] and [10], the correction due to the motion of the Sun (1.5-PN level) can be neglected, while higher corrections (2-PN level) should be used when radio waves are passing close to the Sun (at a few solar radii). Moyer [5], proposed an easy correction to (1) to account for 2-PN effects:

$$
\Delta t=\frac{(1+\gamma) G M}{c^{3}} \ln \left(\frac{R_{1}+R_{2}+R_{12}+\frac{[(G M(1+\gamma)]}{c^{2}}}{R_{1}+R_{2}-R_{12}+\frac{[G M(1+\gamma)]}{c^{2}}}\right)
$$

Equation (4) can be justified in the small impact parameter regime by more comprehensive formulations (e.g. [11] and [12]). Figure 3 shows the net effect on the observables due to the Shapiro delay during the ninth SSC of BepiColombo occurring in June 2023, while Figure 4 shows the difference when the expression (1) is replaced by (4). The latter formulation is adopted here.

The numerical simulations must take into account the limits of the plasma noise compensation procedure. Indeed, due to physical optics effects, there will be a value of the impact parameter (depending on the solar activity), below which the plasma cancellation procedure cannot be applied due to the difficulty in keeping the $\mathrm{X}$ band link. Indeed, between 5 and 8 solar radii the $X$ band signal enters in the strong scintillation regime, where phase measurements become impossible. Even if in practice the data would gradually degrade as the SPE decreases, a simplified approach to account for this effect entails a perfect compensation till a selected, realistic minimum impact parameter, and discarding all data below this limit. In this approach we can take advantage from the results attained during the Cassini experiment, discussed in detail in [13]. Figure 5 shows the tracking passages where the multi-frequency link were available vs the impact parameter: thanks to the 


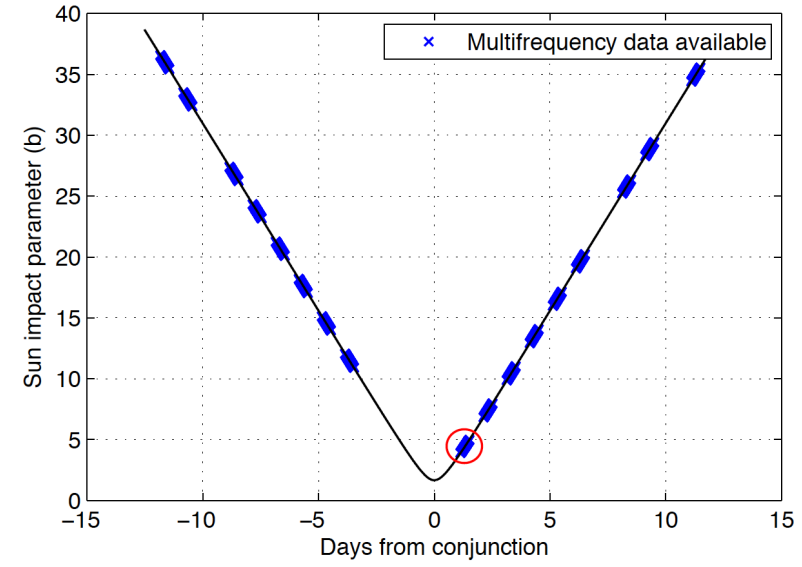

Fig. 5. Tracking passages for the Cassini SCE vs Sun impact parameter. The red circle points out the only non plasma-free passage

plasma noise removal procedure, the plasma-free Doppler observable attained an Allan deviation (ADEV) of $1-2 \cdot 10^{-14}$ at 1000 seconds integration time for all the passages until $\mathrm{b}=7$, while the only passage below $\mathrm{b}=5$ exhibited $\mathrm{a}$ strong degradation (one order of magnitude); following this indication, a nominal limit of seven solar radii is adopted here. However, since the limit depends on many conditions and is actually unpredectable, different values will be considered too.

In our analysis, aimed at assessing the ultimate limits of BepiColombo GR tests, we assume that all SSC are taking place during coast arcs. To optimize the experiment, the spacecraft composite (Solar-electric propulsion mudule, MPO, MMO, solar panels) is assumed to be oriented toward the Sun at constant aspect angle. The preferential attitude is with the solar panels orthogonal to the Sun vector. The effect of the solar radiation pressure force is simulated from a model of the spacecraft diffuse and specular reflectivity ans an acceleration in the radial direction is estimated. Although in principle the non-gravitational accelerations of the spacecraft are measured by a high accuracy accelerometer (ISA), the characteristic frequencies of the relativistic signals $\left(10^{-6}-10^{-5} \mathrm{~Hz}\right)$ are outside the main sensitivity bandwidth of the instrument $\left(10^{-4}-10^{-1} \mathrm{~Hz}\right)$. Rather, microwave tracking may be used to characterize the behaviour of ISA in the low frequency regime.

Both Goldstone and Malargue stations are assumed to provide Doppler and range measurements with the nominal noise until a threshold value of the impact parameter $\left(b_{t}\right)$ of 7. Lastly, for the simulations, we select a single arc of four weeks, two before and two after the conjunction.

We present the results of our analysis under these assumptions and assess the relative uncetainty of $\gamma$ attainable by all ten SSC.

\section{B. Planetary flybys during solar conjunctions}

The superior conjunctions occurring close to a flyby need further considerations. As reported in Table 1, the first SSC will occur just before a Venus fluyby. However, this flyby

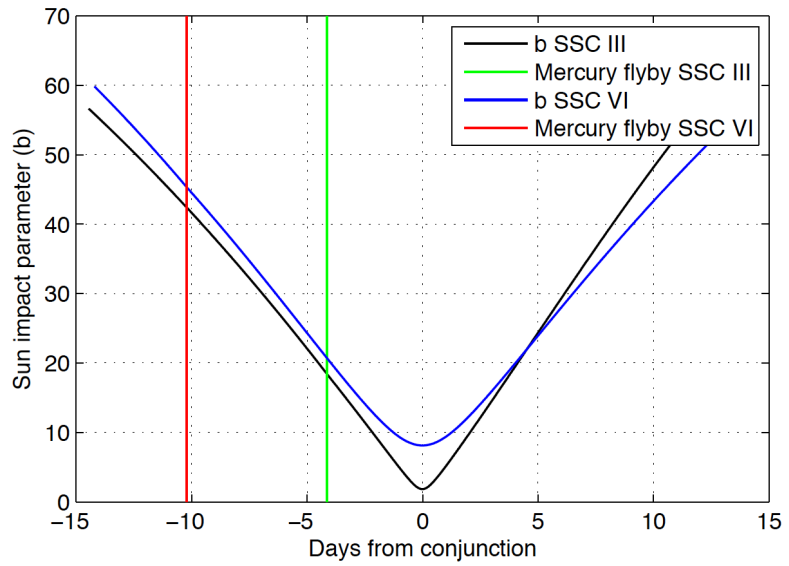

Fig. 6. Flybys positions during the third and sixth SSC

will happen on the September 22, 2019, eleven days after the end of tracking period (on September 11). Errors in the gravitational parameter and in the orbit of Venus have undetectable effects on the estimation of the relativistic delay. In contrast, the third and the sixth SSC include a flyby in the observation period, as shown in Figure $6^{3}$. Those flybys require a careful analysis, as the proximity of a massive third body strongly affects the spacecraft trajectory. Uncertainties in the planet's ephemeris and gravity field need to be accounted for in the analysis. Failing to do so lead to unrealistic small covariances.

Since the flybys involve the planet Mercury, we can take advantage of the estimates from NASA's MESSENGER mission. The Hermean harmonic coefficients are already available in [14], but further improvements are expected from the analysis of the final phases of the mission. At the end of BepiColombo mission, an even more accurated gravity field will be available, together with a better estimate of the ephemeris of the planet. Today the orbit of Mercury is known with an accuracy of hundred of meters [15].

\section{Results}

Table 2 summarizes the results of our analysis. In an optimal configuration, all the SSC would be able to improve the Cassini's estimate of $\gamma$, mainly because range measurements and a second $\mathrm{Ka}$ band tracking station (at Malargue, Argentina) are available. Differences in the estimate of $\gamma$ are within a factor of about 2 for all SSC, with best

${ }^{3}$ For details on the flybys see [8]

TABLE II. SIMULATIONS RESUlts $\left(b_{t}=7\right)$

\begin{tabular}{cccccc}
\hline SSC & I & II & III & IV & V \\
\hline \hline $\boldsymbol{\sigma}_{\boldsymbol{\gamma}}\left(\cdot 10^{-6}\right)$ & 10.4 & 7.82 & 9.19 & 6.14 & 5.07 \\
\hline
\end{tabular}

\begin{tabular}{cccccc}
\hline SSC & VI & VII & VIII & IX & X \\
\hline \hline $\boldsymbol{\sigma}_{\boldsymbol{\gamma}}\left(\cdot 10^{-6}\right)$ & 6.28 & 7.87 & 5.03 & 4.26 & 6.51 \\
\hline
\end{tabular}



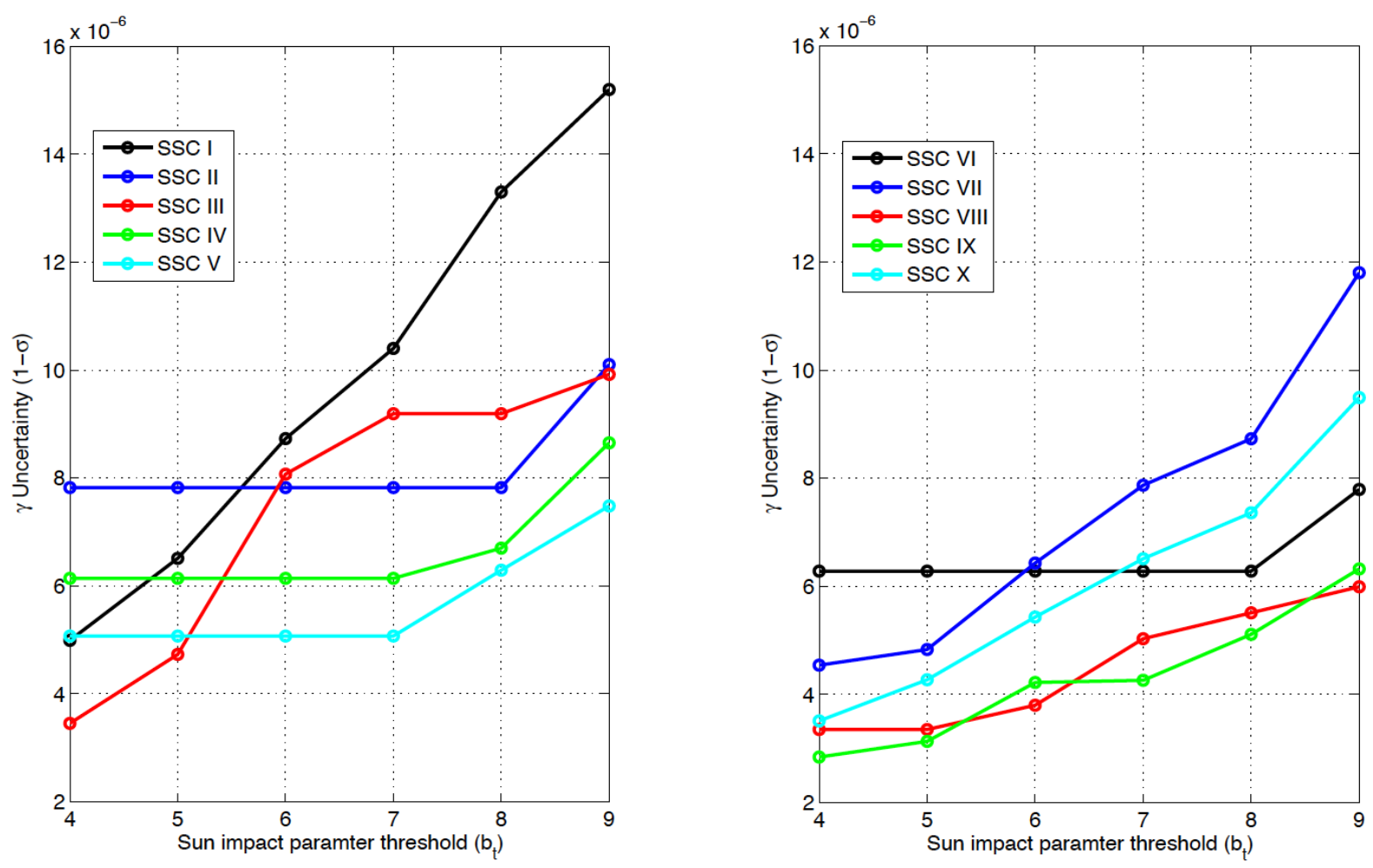

Fig. 7. Results for $\gamma$ determination as a function of the applicability conditions for the plasma noise removal process $\left(b_{t}\right)$

results found in the eighth and ninth SSC.

Non-gravitational accelerations (mostly due to the solar radiation pressure) are estimated at level of $10^{-14} \mathrm{~km} / \mathrm{s}^{2}$, the same level of accuracy attained in the estimation of the accelerations due to the radioisotope thermal generator in the Cassini experiment [13].

In the hypothesis that the ephemeris and the gravity field of Mercury were perfectly known, we obtain an estimation of $\gamma$ with an uncertainty at level of $8.84 \cdot 10^{-7}$ and $1.61 \cdot 10^{-6}$ respectively for the third and the sixth SSC. These outstanding (but unrealistic) results are due to the favorable effect of a flyby on the trajectory. Indeed, the rapidly varying dynamics of the spacecraft strongly constrains the spacecraft trajectory. However, as discussed in the previous section, we must deal with the uncertainties in the orbit of the planet and its gravity field. Although MESSENGER largely improved our knowledge about Mercury, the current uncertainties in its cahracteristics are still able to degrade the solution and the determination of $\gamma$. The estimate of this parameter would be affected by a significant systematic error. The error may be canceled out by including in the solve-for parameters the ephemeris of the planet and the coefficients of the gravity field

TABLE III. MULTI-ARC SIMULATIONS RESUlTS $\left(b_{t}=7\right)$

\begin{tabular}{cccccc}
\hline SSC & VIII+IX & VI+IX & I+III+VI & I+III+VI+IX & All \\
\hline \hline $\boldsymbol{\sigma}_{\boldsymbol{\gamma}\left(\cdot 10^{-6}\right)}$ & 3.24 & 3.27 & 4.45 & 2.96 & 1.93 \\
\hline
\end{tabular}

to the required degree and order. This approach provides a solution at the cost of some degradation in the determination of $\gamma$. Our simulations indicate that for both the SSC of interest, the additional parameters may degrade the solution for $\gamma$ at the same level of what is reached by considering the SSC starting just after the flyby. For this reason, in Table 2, the tracking periods in the third and sixth SSC begin after the closest approach (see Figure 6).

Figure 7 reports on the results obtained by varying the threshold impact parameter $b_{t}$. The best opportunities to test GR are in the second part of the cruise, and the eighth and ninth SSC are the most promising opportunities independly of $b_{t}$. Obvioulsy, the use of several SSC in a combined solution results in a significant reduction of the covariances (see Table 3). Since the best SSC belong to thrust arcs, where the solar electric propulsion is activated (continuously during the eighth, partially over the ninth one), we focus mainly on the results obtained by combining one them (the ninth one) with those already scheduled to be thrust-free.

TABLE IV. IX SSC CONTINGENCIES RESULTS $\left(b_{t}=7\right)$

\begin{tabular}{ccccc}
\hline & Nominal & - Malargue & + Madrid & 20 Days \\
\hline \hline $\boldsymbol{\sigma}_{\boldsymbol{\gamma}}\left(\cdot 10^{-6}\right)$ & 4.26 & 5.26 & 3.50 & 5.42 \\
\hline \hline & Nominal & $\mathbf{2}$ x Noise & Only Doppler & Only Range \\
\hline \hline $\boldsymbol{\sigma}_{\boldsymbol{\gamma}\left(\cdot 10^{-6}\right)}$ & 4.26 & 8.12 & 6.95 & 5.89 \\
\hline
\end{tabular}


Lastly, in Table 4 we report some additional results when the ninth SSC is analysed under the following contingencies: 1) only DSS 25 Goldstone is available (no support from the ESTRACK station in Malargue); 2) the use of a third station in Spain with the multi-frequency capability; 3) a reduced tracking period (twenty days instead of four weeks); 4) a noise twice the nominal one; 5) only Doppler/range measurements available. The results show the importance of an effective reduction of the noise by means of the multi-frequency system $^{4}$.

\section{CONCLUSIONS}

Our work provides an assessment of the cruise tests of GR to be carried out by the mission BepiColombo during its cruise phase to Mercury. The use of multi-frequency radio link both for Doppler and range observables, combined with the potential use of an additional ground station at Malargue, Argentina, will provide an estimate of the PN parameter $\gamma$ with substantial improvements over the current accuracies. In the optimal configuration, $\gamma$ may be determined at level of $5 \cdot 10^{-6}$. Further improvements at this result can be attained in case of favourable conditions for the plasma noise compensation procedure provided by the multi-frequency link or by combining different conjunctions in a global solution.

The predicted results on the determination of $\gamma$, will be also used as an a priori input for testing GR during the orbital phase around Mercury. These additional opportunities are expected to further improve the accuracy in the determination of $\gamma$ as well as the determination of other PN and physical parameters [16].

\section{REFERENCES}

[1] B. Bertotti, L. Iess and P. Tortora, A test of general relativity using radio links with the Cassini spacecraft, Nature 425, 374-376 (2003).

[2] A. Milani, D. Vokrouhlicky, D. Villani, C. Bonanno and A. Rossi, Testing general relativity with the BepiColombo radio science experiment, Phys. Rev. D 66, 0820012002.

[3] I.I. Shapiro, Fourth test of general relativity, Phys. Rev. Lett. 13, 789-791 (1964).

[4] C.M. Will, Theory and experiment in gravitational physics, Cambridge University Press, Cambridge (1993).

[5] T.D. Moyer, Formulation for observed and computed values of deep space network data types for navigation, Wiley-Interscience, New York (2003)

[6] L. Iess, G. Giampieri, J.D. Anderson and B. Bertotti, Doppler measurements of the solar gravitational deflection, Class. Quant. Grav. 16, 1487-1502 (1999).

[7] L. Iess, G. Boscagli, Advanced radio science instrumentation for the mission BepiColombo to Mercury, $\quad$ Pla. Sp. Sci. 49,1597-1608 (2001).

[8] R. Jehn, BepiColombo Mercury cornerstone consolidated report on mission analysis, MAS working paper no 525, ESOC, Dardstadt, Germany, (2014).

[9] A. Milani, G. Tommei, D. Vokrouhlicky, E. Latorre and S. Cicalo', relativistic model for the BepiColombo radioscience experiment, In Klioner, S., Seidelman, P.K., Soffel, M. (eds) Relativity in Fundamental Astronomy: Dynamics, Reference Frames, and Data Analysis, IAU Symposium 261, 356-365 (2010).

[10] G. Tommei, A. Milani and D. Vokrouhlicky, Light-time computations for the BepiColombo radio science experiment, Celest. Mech. Dyn. Astr. 107:285-298, (2010).

\footnotetext{
${ }^{4}$ Obviously not only the plasma noise must be calibrated but also the noise introduced by the troposphere, as described in [13]
}

[11] N. Ashby, B. Bertotti, Accurate light-time correction due to a gravitating mass, ArXiv e-prints 0912.2705, (2009).

[12] P. Teyssandier, C. Le Poncin-Lafitte, General post-Minkowskian expansion of time transfer functions, Class. Quantum Grav. 25, 145020 (2008)

[13] P. Tortora, L. Iess,J.J. Bordi,J.E. Ekelund and D.C. Roth, Precise Cassini navigation during solar conjunctions throuh multifrequency plasma calibrations, Juornal of guidance, Control, and Dynamics 27, No.2 (2004)

[14] http://pds-geosciences.wustl.edu/messenger/mess-h-rss_mla-5-sdpv1/messrs_1001/data/shadr/ggmes_50v06_sha.lbl

[15] E. Mazarico et al. The gravity field, orientation, and ephemeris of Mercury from MESSENGER observations after three years in orbit, journal of Geophysical Research, Vol. 119, Issue 12, 2417-2436, (2014)

[16] G. Schettino, S. Cicalo', S. Di Ruzza and G. Tommei, The relativity experiment of MORE: global full-cycle simulation and results, in Proceedings of 2nd IEEE International Workshop on Metrology for Aerospace, Special Session on Relativistic Metrology, Benevento, Italy (2015) 